\title{
Global Structure of Low-Latitude and Equatorial Geomagnetic Pulsations Associated with Storm Sudden Commencements
}

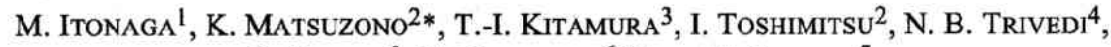 \\ R. HORITA ${ }^{5}$, T. WATANABE ${ }^{6 * *}$, and J. RIDDICK ${ }^{7}$ \\ ${ }^{1}$ Computation Center, Kyushu University, Higashi-ku, Fukuoka 812, Japan \\ ${ }^{2}$ Department of Physics, Kyushu University, Higashi-ku, Fukuoka 812, Japan \\ ${ }^{3}$ Department of Earth and Planetary Sciences, Kyushu University, Higashi-ku, Fukuoka 812, Japan \\ ${ }^{4}$ Instituto Nacional de Pesquisas Espaciais, São José dos Campos, Brazil \\ ${ }^{5}$ Department of Physics and Astronomy, University of Victoria, Victoria, B.C., Canada \\ ${ }^{6}$ Department of Geophysics and Astronomy, University of British Columbia, Vancouver, B.C., Canada \\ ${ }^{7}$ British Geological Survey, Edinburgh, U.K.
}

(Received September 8, 1994; Revised February 10, 1995; Accepted March 24, 1995)

Using geomagnetic data from a global network equipped with fluxgate magnetometers and data loggers by Kyushu University, two moderate storm sudden commencement (SSC) events which occurred on Jun. 10 and Aug. 13, 1992 were analyzed. The network consists of about 30 stations, the main portion of which are arrayed nearly along three magnetic meridians (Japanese, Brazilian and African meridians) in low-latitude and equatorial regions. As for low-latitude and equatorial SSC-associated geomagnetic pulsations (Psc's), the following results were obtained. (1) The shape of the waveform is consistently ordered by local time for all low-latitude and equatorial stations; it is steeper near noon (or near the source) and has a more moderate slope near midnight (far from the source). This indicates that the Psc's are not cavity-mode oscillations but compressional hydromagnetic (HM) waves propagating from dayside to nightside two-dimensionally along the equatorial plane. (2) The Psc's have globally almost identical dominant frequencies which agree well with those of Pi2's. This implies that the frequencies of lowlatitude and equatorial pulsations may be determined by the global structure of the magnetosphere or the plasmasphere independently of source mechanisms. During its passage through the plasmapause the compressional HM wave generated in the outer magnetosphere might be regulated in such a waveform as could be expressed as a superposition of exponentially damped sinusoids at ground stations, whose frequencies are globally almost identical.

\section{Introduction}

It is well known that damped-type geomagnetic pulsations are excited by storm sudden commencements (SSC's) and sudden impulses (SI's). These pulsations have been observed from high latitudes to low latitudes on the ground (Saito and Matsushita, 1967) and also near the equatorial plane in the magnetosphere by satellites (Kaufmann and Walker, 1974). Such SSC-associated pulsations were named Psc's and classified into four groups by Saito and Matsushita (1967) according to period ranges in a similar way as Pc pulsations, i.e., Psc 1 (0.2-5 s), Psc 2.3 (5-30 s), Psc 4 (30-150 s) and Psc 5 (150-600 s). They found that Psc 2.3's are generally observed at middle and low latitudes, while Psc 4 's and Psc 5 's are observed in the subauroral and auroral regions. Recently, Wedeken et al. (1986) showed through a case study of an SSC with a number of magnetometers and the GEOS 2 satellite that the Psc had a complex spatial variation of spectral structure with a small value $(m=0 \sim 4)$ of the azimuthal wavenumber, some features of which were consistent with the coupled global mode theory (Kivelson and Southwood, 1985, 1986). Further,

*Now at Ishikawajima System Technology Co., Ltd., Shinagawa-ku, Tokyo 141, Japan.

**N Now at Department of Communications Engineering, Tohoku Institute of Technology, Taihaku-ku, Sendai 982, Japan. 
using fluxgate magnetometer data from the coordinated observatories along the $210^{\circ}$ magnetic meridian, Yumoto et al. (1992) found that an intense SSC excited cavity-mode Pc 3 pulsations with duration less than $20 \mathrm{~min}$ and identical 15.5 and $25.3 \mathrm{mHz}$ frequencies in a low-latitude region from $L=1.14$ to 2.13 .

In the present paper, using geomagnetic data from a global network equipped with fluxgate magnetometers and data loggers by Kyushu University, we shall show that Psc's are not cavity-mode oscillations but propagating waves. We analyzed two moderate SSC events which occurred on Jun. 10 and Aug. 13, 1992. Raw geomagnetic data are smoothed by a one-pass method with a piecewise cubic polynomial (Itonaga and Kitamura, 1994) and Psc's are extracted from the raw data as residuals. Then, the Psc is regarded as a superposition of exponentially damped sinusoids and the parameters (frequency, damping factor, initial amplitude and initial phase) of the damped sinusoids are estimated using a modified Pisarenko method (Itonaga, 1990). We will examine the global structure of low-latitude and equatorial Psc's on the basis of the comparison between waveforms and the parameter estimation, and will consider an origin of the global structure from a standpoint of propagating wave.

\section{Data}

The data were obtained from a global geomagnetic network equipped with fluxgate magnetometers and data loggers by Kyushu University. The network consists of about 30 stations, the main portion of which are arrayed nearly along three magnetic meridians (Japanese, Brazilian and African meridians) in low-latitude and equatorial regions. The coordinates of the stations used in the present study are given in Table 1 . The clock of the data logger is automatically calibrated every $5 \mathrm{~min}$ by VLF (Omega) signals, so that the accuracy of the clock is always kept within $\pm 0.1 \mathrm{~s}$. The analogue output signals of the $H, D$ and $Z$ components from the magnetometer were sampled every $3 \mathrm{~s}$. The sampled data were differentiated and each resulting value was stored with precision of 8 bits. Since too rapid changes of the $H$ component signal in intense SSC's could cause overflows in the representation of differentiated data with precision of 8 bits, two moderate SSC events listed in Table 2 were selected for the analysis. The start time of each SSC event and the number of stations used in the analysis of each event are also given in Table 2.

Table 1. Coordinates of stations used in the study.

\begin{tabular}{lllrrrrr}
\hline Station & Abbr. & Nation & \multicolumn{2}{c}{ Geographic } & \multicolumn{2}{c}{ Geomagnetic } & Dip \\
\hline Kuju & KUJ & Japan & $33.1^{\circ}$ & $131.2^{\circ}$ & $22.8^{\circ}$ & $200.0^{\circ}$ & $46.7^{\circ}$ \\
Guam & GUA & U.S.A. & $13.6^{\circ}$ & $144.9^{\circ}$ & $4.6^{\circ}$ & $214.8^{\circ}$ & $11.2^{\circ}$ \\
Darwin & DAR & Australia & $-12.4^{\circ}$ & $130.8^{\circ}$ & $-22.6^{\circ}$ & $203.1^{\circ}$ & $-40.9^{\circ}$ \\
Victoria & VIC & Canada & $48.5^{\circ}$ & $236.6^{\circ}$ & $54.3^{\circ}$ & $295.7^{\circ}$ & $70.5^{\circ}$ \\
Sao Luis & SLZ & Brazil & $-2.4^{\circ}$ & $315.9^{\circ}$ & $7.5^{\circ}$ & $27.0^{\circ}$ & $3.1^{\circ}$ \\
Eusebio & EUS & Brazil & $-3.5^{\circ}$ & $321.7^{\circ}$ & $5.8^{\circ}$ & $32.8^{\circ}$ & $-5.8^{\circ}$ \\
Teresina & TER & Brazil & $-5.0^{\circ}$ & $317.5^{\circ}$ & $4.7^{\circ}$ & $28.4^{\circ}$ & $-3.5^{\circ}$ \\
Santa Maria & SMA & Brazil & $-29.4^{\circ}$ & $306.6^{\circ}$ & $-18.9^{\circ}$ & $16.0^{\circ}$ & $-31.0^{\circ}$ \\
Hartland & HAR & U.K. & $51.0^{\circ}$ & $355.5^{\circ}$ & $54.2^{\circ}$ & $80.3^{\circ}$ & $66.2^{\circ}$ \\
Tamanrasset & TAM & Algeria & $22.8^{\circ}$ & $5.5^{\circ}$ & $24.9^{\circ}$ & $81.2^{\circ}$ & $27.7^{\circ}$ \\
\hline
\end{tabular}

Table 2. SSC events analyzed in the study.

\begin{tabular}{ccc}
\hline Date & Time (UT) & Number of stations \\
\hline Jun. 10,1992 & $04: 03$ & 10 \\
Aug. 13, 1992 & $15: 15$ & 7 \\
\hline
\end{tabular}


Jun. 10, 1992 II-comp. (raw) $30 \mathrm{nT} / \mathrm{div}$

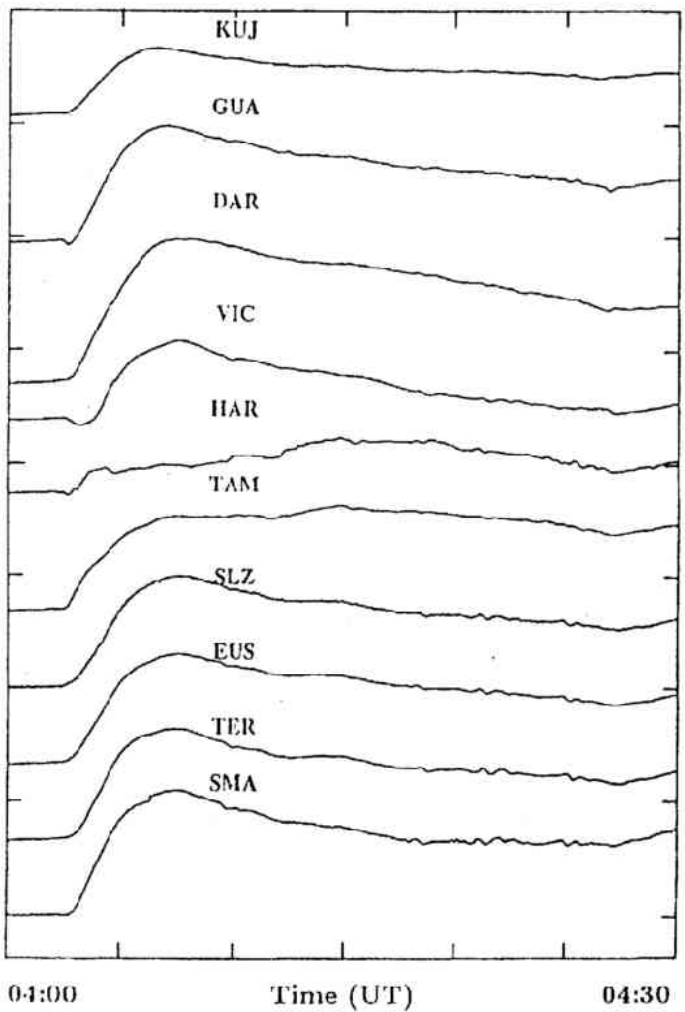

Jun. 10, 1992 D-comp. (raw)

$30 \mathrm{nT} / \mathrm{div}$

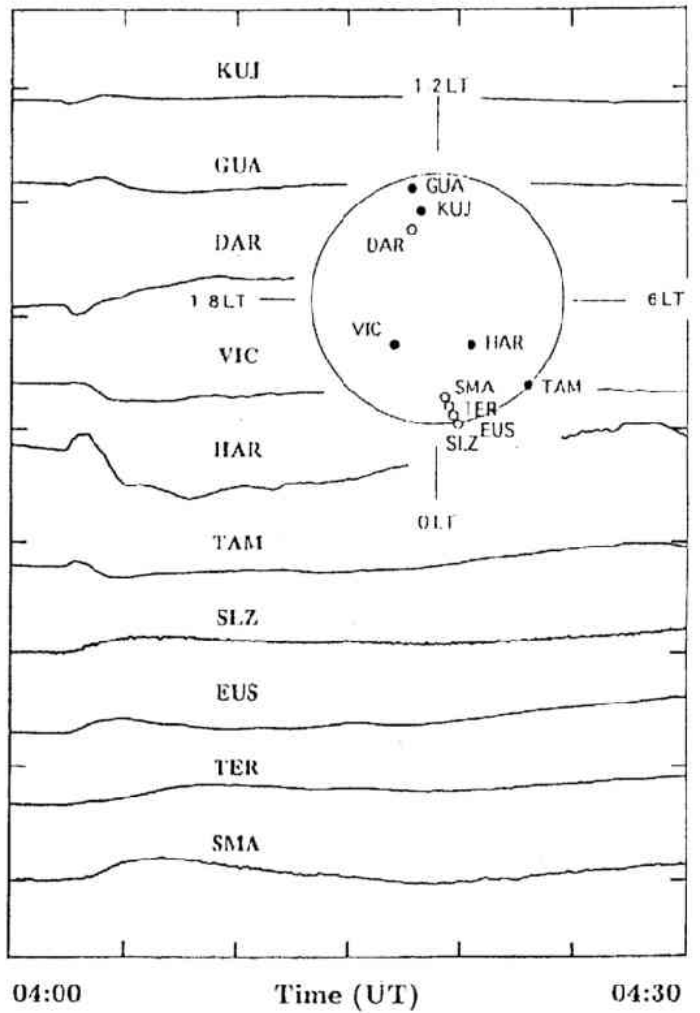

Fig. 1. Raw $H$ and $D$ component data recorded on Jun. 10, 1992. A moderate SSC began at around 04:03 UT. The locations of stations are also illustrated with solid and open circles corresponding to the northern and southern stations, respectively.

Aug. 13, 1992 H-comp. (raw)

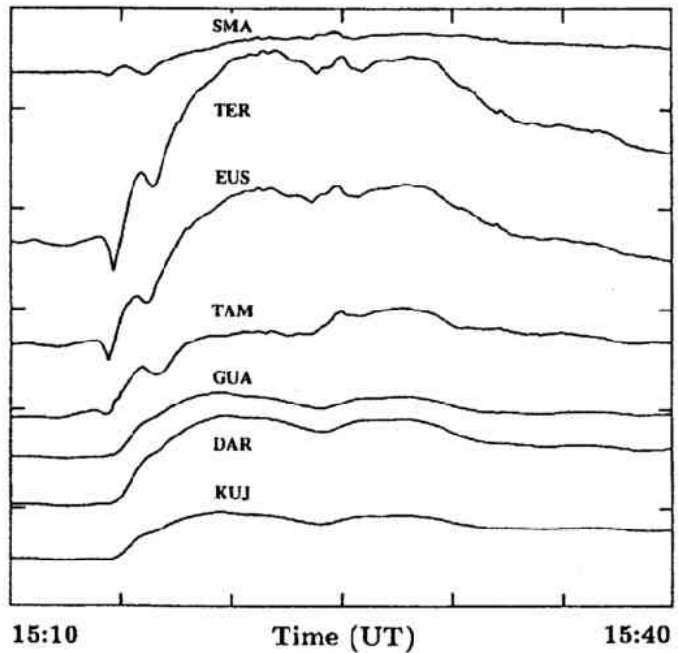

Aug. 13, 1992 D-comp. (raw)

$30 \mathrm{nT} / \mathrm{div}$

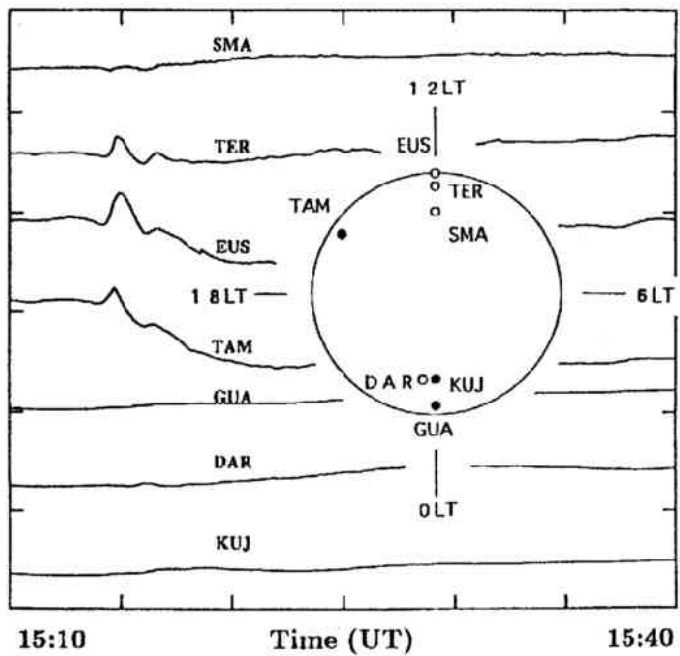

Fig. 2. Raw $H$ and $D$ component data recorded on Aug. 13, 1992. A moderate SSC began at around 15:15 UT. The locations of stations are also illustrated with solid and open circles corresponding to the northern and southern stations, respectively. 
Figure 1 shows raw $H$ and $D$ component data recorded on Jun. 10,1992. It is found that a moderate SSC began at around 04:03 UT. In this figure the locations of stations are also illustrated with solid and open circles corresponding to the northern and southern stations, respectively. Also displayed in Fig. 2 are raw $H$ and $D$ component data recorded on Aug. 13, 1992. It is found that a moderate SSC began at around 15:15 UT. During the SSC event on Jun. 10, 1992, the Japanese meridian including KUJ, GUA and DAW was located near noon and the Brazilian meridian including SLZ, EUS, TER and SMA near midnight. On the other hand, the locations of the Japanese and Brazilian meridians are reversed during the SSC event on Aug. 13, 1992.

Since our research interests are in low-latitude and equatorial Psc's and it is thought that features of such pulsations appear primarily in their $H$ component variations, nothing will be said about the Psc's at highest latitude stations VIC and HAR and also about their $D$ component variations.

\section{Waveform of Psc's}

Since we need to extract Psc's as in a pure state as possible to perform a direct comparison between their waveforms, we make use of a method for fitting a piecewise cubic polynomial (PCP) to a sequence of geomagnetic data containing oscillations such as Psc's superposed on rapid background changes such as main impulses (MI's) of SSC's, which has been presented by Itonaga and Kitamura (1994). Such a

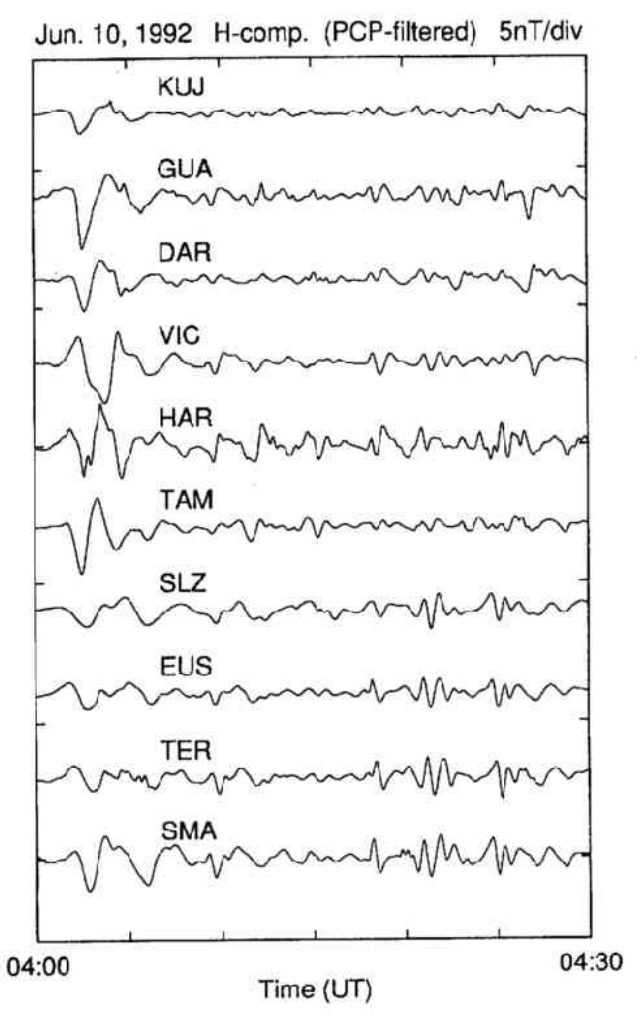

Fig. 3.

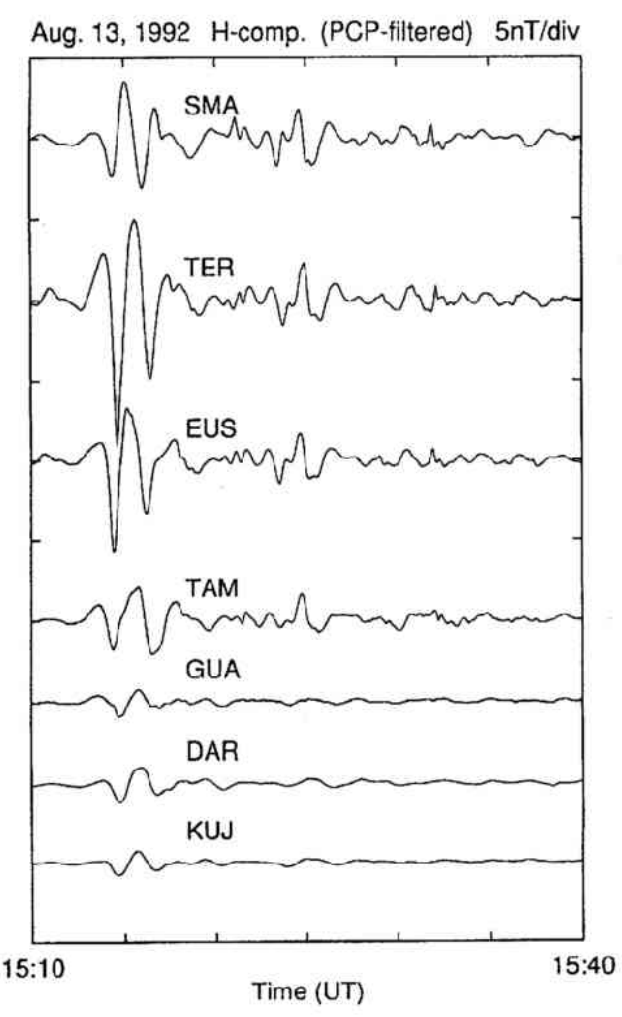

Fig. 4.

Fig. 3. Psc's extracted by processing the raw $H$ component data in Fig. 1 with the PCP filter of $\mu=7$. The value of $\mu$ corresponds to the cutoff frequency of $5.27 \mathrm{mHz}$ for $3 \mathrm{~s}$ sampling.

Fig. 4. Psc's extracted by processing the raw $H$ component data in Fig. 2 with the PCP filter of $\mu=7$. The value of $\mu$ corresponds to the cutoff frequency of $5.27 \mathrm{mHz}$ for 3 s sampling. 
fitting method corresponds to a nonlinear lowpass filter and is named a PCP filter (Itonaga, 1995). The PCP filter is noncausal, and it practically brings no phase shift onto the output and residual data so far as the input data has no dominant components of which frequencies lie within its transition band. While the PCP filter is accompanied with a parameter $\mu$ determing the degree of smoothing, the relation between $\mu$ and its cutoff frequency has been given in Itonaga (1995). The PCP filter preserves rapid background changes such as MI's of SSC's in the output data much better than classical linear lowpass filters, and so the Psc's can be extracted as residuals with little deformation using this filter. Figures 3 and 4 are results obtained by processing the raw $H$ component data in Figs. 1 and 2 with the PCP filter of $\mu=7$, respectively, and show Psc's. This value of $\mu$ corresponds to the cutoff frequency of $5.27 \mathrm{mHz}$ for $3 \mathrm{~s}$ sampling.

Displayed in Fig. 5 are superpositions of $H$ component traces of the Psc's in the Brazilian (noon) and Japanese (midnight) meridians on Aug. 13, 1992. This figure gives us an impression that the shape of the waveform is consistently ordered by local time for all low-latitude and equatorial stations; it is steeper near noon (or near the source) and has a more moderate slope near midnight (far from the source). The Psc's have quite similar waveforms in the Japanese meridian. Similar waveforms are also found for the Psc's

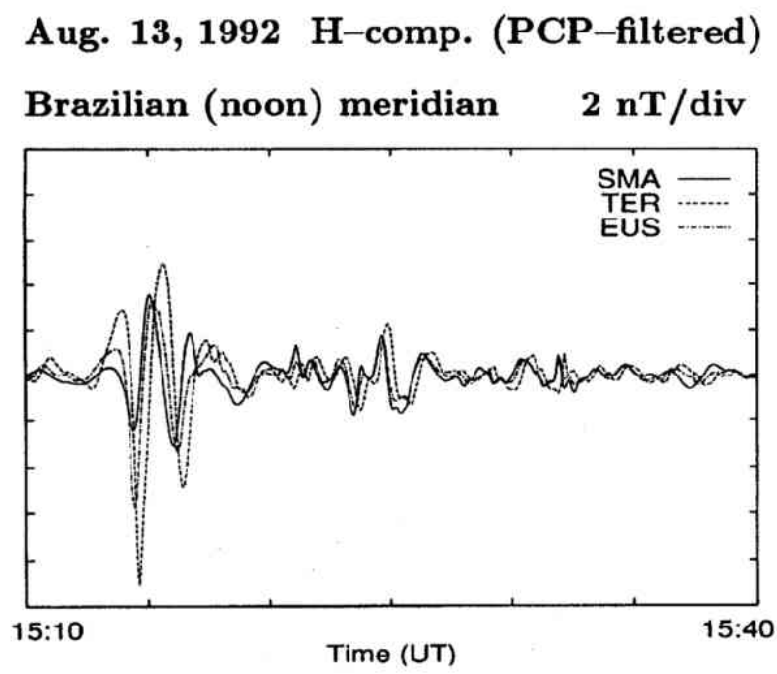

Japanese (midnight) meridian $2 \mathrm{nT} / \mathrm{div}$

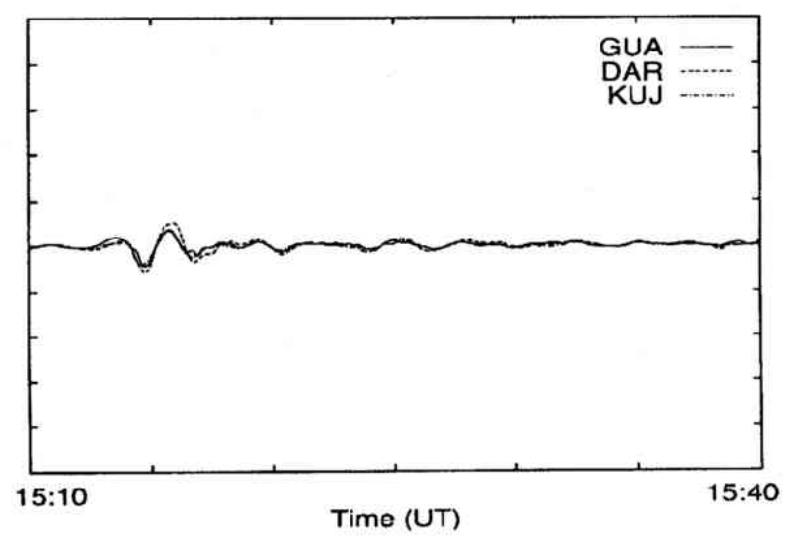

Fig. 5. Superpositions of $H$ component traces of the Psc's in the Brazilian (noon) and Japanese (midnight) meridians on Aug. $13,1992$. 
in the Brazilian meridian, although the negative kick at around 15:15 UT has a larger amplitude nearer the dip equator and a time delay is clearly seen at TER. However, the negative kick is thought to be not a part of the Psc but the preliminary reverse impulse (PRI). The PRI and the time delay at TER, which appears to be located nearer the dip equator than EUS and SMA (cf. Table 1), will be due to the enhanced ionospheric conductivity at the dayside dip equator (see Discussion). Then, to avoid the influence of the enhanced ionospheric conductivity on Psc's, we compare the waveforms observed at off-equatorial stations. Figure 6 shows superpositions of $H$ component traces of the Psc's at EUS and GUA and at SMA and DAR. The above impression is also confirmed from this figure. Further, it is found that the phase of the Psc in the Japanese (midnight) meridian is delayed compared with that in the Brazilian (noon) meridian, although the phase difference is ambiguous except immediately after the SSC owing to the moderate waveform in the Japanese meridian.

It is difficult to interpret the global structure of the Psc's mentioned above from a standpoint of cavitymode oscillation. From a standpoint of propagating wave, on the other hand, the moderate waveform near midnight may be due to a kind of diffraction effect that among compressional hydromagnetic(HM) waves which have entered the plasmasphere, those waves with lower frequencies (or longer wavelengths)

Aug. 13, 1992 H-comp. (PCP-filtered)

$$
2 \mathrm{nT} / \mathrm{div}
$$

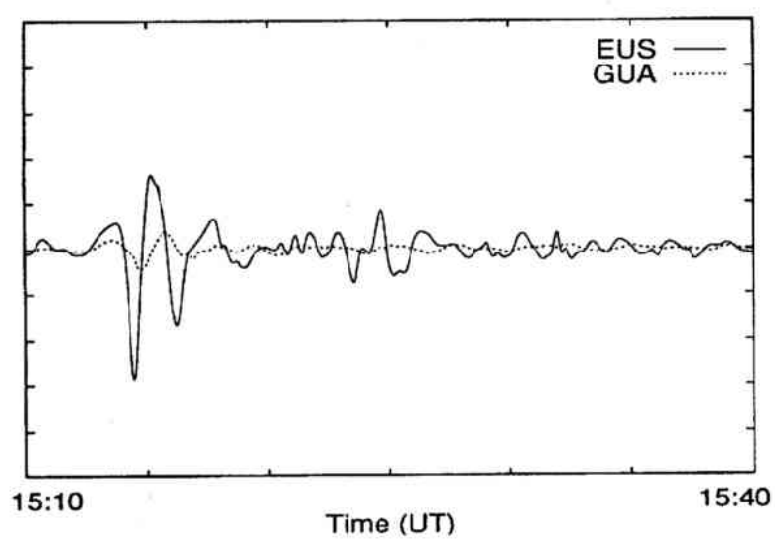

$2 \mathrm{nT} / \mathrm{div}$

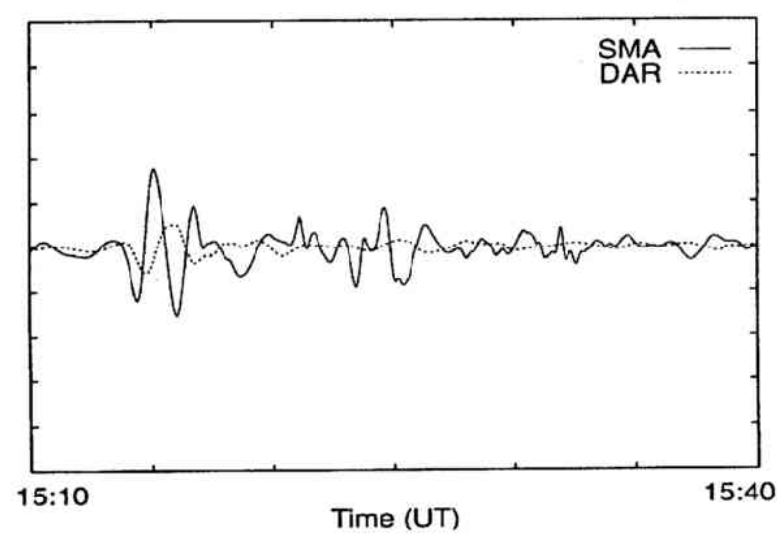

Fig. 6. Superpositions of $H$ component traces of the Psc's at EUS and GUA and at SMA and DAR on Aug. 13, 1992. 
propagate around the earth to midnight with less attenuation (cf. Fig. 10 of Wilken et al. (1982)). Further, the standpoint of propagating wave is also supported by the fact that the phase of the Psc near midnight is delayed compared with that near noon. Thus, we contend that the low-latitude and equatorial Psc's are not cavity-mode oscillations but compressional HM waves propagating from dayside to nightside twodimensionally along the equatorial plane. Here, it should be noted that although the phase difference between the Psc's near noon and near midnight suggests the propagation of HM wave from dayside to nightside, it is not always consistent with the traveling time of HM wave near the earth in the magnetosphere because the induced current in the intermediate ionosphere would prevent the HM wave from entering the atmosphere and so the apparent phase difference on the ground would become smaller compared with the traveling time of HM wave (see Discussion). The apparent phase difference on the ground might decrease with decreasing latitude (Ohnishi and Araki, 1992).

In case of the SSC event on Jun. 10, 1992, although the global structure of the Psc's is ambiguous owing to their irregular waveforms (Fig. 7), it is also found in Fig. 8 displaying a superposition of $H$ component traces of the Psc's at off-equatorial stations EUS and GUA that the waveform is steeper at GUA

Jun. 10, 1992. H-comp. (PCP-filtered)

Japanese (noon) meridian $1 \mathrm{nT} / \mathrm{div}$

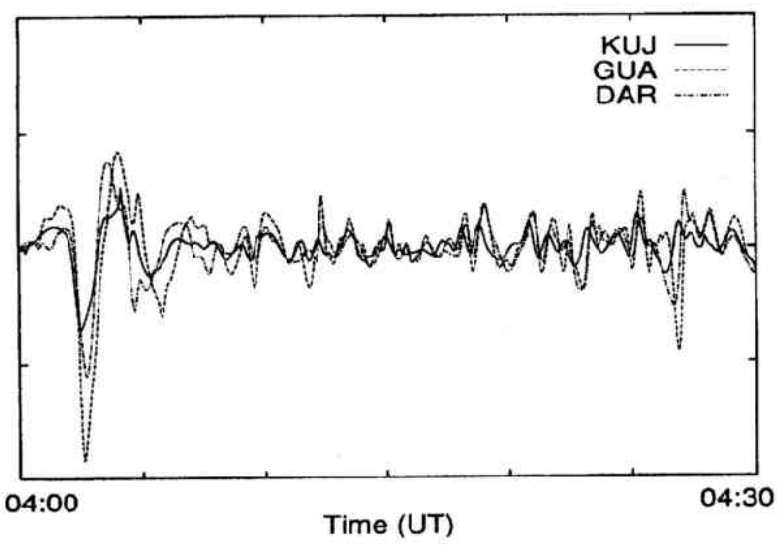

Brazilian (midnight) meridian $1 \mathrm{nT} /$ div

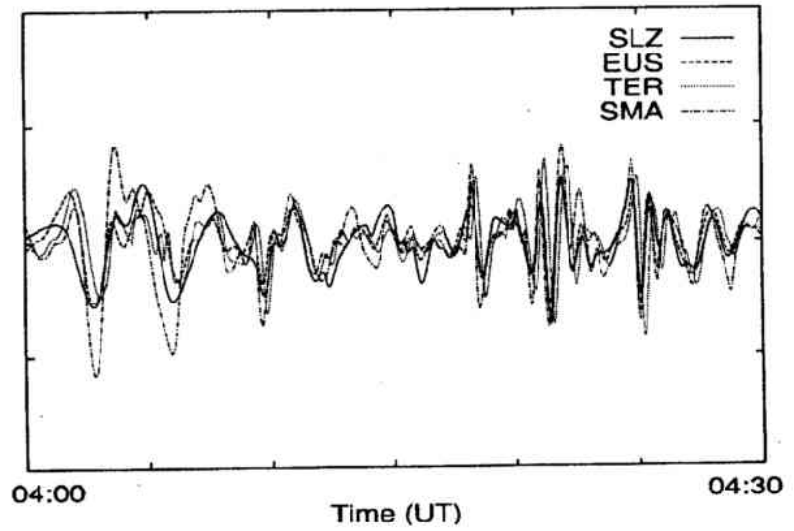

Fig. 7. Superpositions of $H$ component traces of the Psc's in the Japanese (noon) and Brazilian (midnight) meridians on Jun. 10, 1992. 
Jun. 10, 1992 H-comp. (PCP-filtered)

$1 \mathrm{nT} / \mathrm{div}$

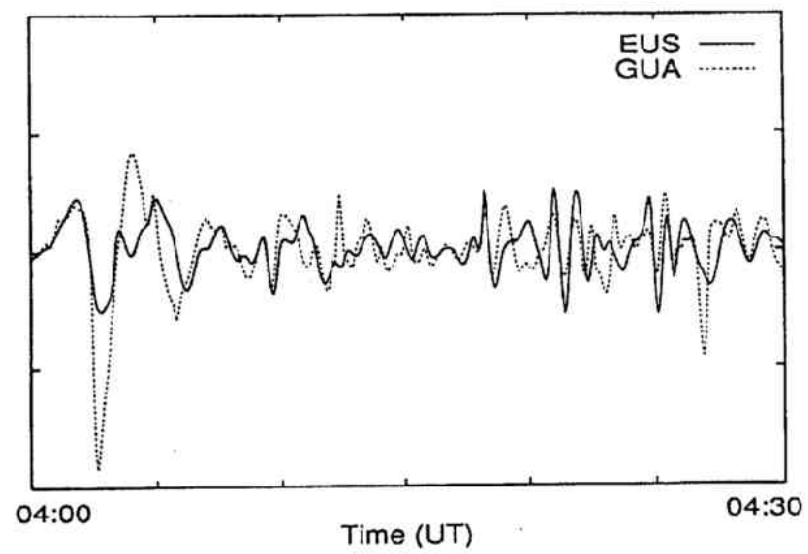

Fig. 8. Superposition of $H$ component traces of the Psc's at EUS and GUA on Jun. 10, 1992.

near noon and has a moderate slope at EUS near midnight, and the phase at EUS is delayed compared with that at GUA. Then, we can also conclude that the low-latitude and equatorial Psc's are not cavity-mode oscillations but compressional HM waves propagating from dayside to nightside. It should be noticed that the oscillations starting at around 04:18 UT are Pi2's having their source on the nightside in the magnetosphere and are larger in magnitude at EUS near midnight than at GUA near noon (Fig. 8). If the compressional $\mathrm{HM}$ wave propagates two-dimensionally along the equatorial plane, it will be expected that the $H$ component of the ground magnetic field produced by the HM wave becomes smaller in magnitude as the latitude increases. However, the Psc at SMA has an anomalously large amplitude and its amplitude is comparable to or larger than that at DAR (Fig. 7). This implies that the Psc at SMA is not directly produced by the compressional HM wave propagating two-dimensionally along the equatorial plane but is due to some other propagation modes. Thus, a direct comparison between waveforms at SMA and DAR will be meaningless in this case and so the corresponding superposition of $H$ component traces is not shown in Fig. 8.

It is reported that the enhancement of the SSC amplitude during midday hours is larger in the American equatorial region than in any other equatorial regions (Rastogi, 1993). Then, when the Brazilian meridian is located near noon and the Japanese meridian near midnight, the impression that the waveform is steeper near noon and has a moderate slope near midnight may be emphasized owing to the larger midday enhancement of the SSC amplitude in the American equatorial region. However, since our contention that the low-latitude and equatorial Psc's are not cavity-mode oscillations but propagating HM waves is based on the comparison between Psc waveforms at off-equatorial stations, there will be no reason why such a midday enhancement forces it to be altered.

\section{Parameter Estimation of Psc's}

It serves the purpose of understanding their global structure more deeply to examine what frequency component the Psc's comprise. Then, assuming a model

$$
s_{n}=\sum_{k=1}^{p} A_{k} \mathrm{e}^{-\alpha_{k} n \Delta t} \cos \left(\omega_{k} n \Delta t+\phi_{k}\right) \quad(n=0,1, \ldots, N-1)
$$


for a $5 \min (N=100)$ interval of the residual data obtained using the PCP filter, where $\Delta t(=3 \mathrm{~s})$ is the sampling interval, we estimated the parameters (damping factors $\alpha_{k}$ 's, angular frequencies $\omega_{k}$ 's, initial amplitudes $A_{k}$ 's and initial phases $\phi_{k}$ 's) in each interval using a modified Pisarenko method (Itonaga, 1990) while shifting the interval by $15 \mathrm{~s}$. Although the modified Pisarenko method is inferior in estimation ability to the maximum likelihood (ML) method in which the variance of estimation error reaches the CramerRao lower bound, it is one of the methods with the highest performance except for the ML method. In addition, this method is much superior in computational cost to the ML method. The number $p$ of exponentially damped (or growing) sinusoids was determined using the minimum description length (MDL) (Rissanen, 1978). In the present case the MDL is given by

$$
\mathrm{MDL}=\frac{N}{2} \ln \hat{\sigma}^{2}+2 p \ln N,
$$

where constant terms are neglected and $\hat{\sigma}^{2}$ is calculated by

$$
\hat{\sigma}^{2}=\frac{1}{N} \sum_{n=0}^{N-1}\left|r_{n}-\hat{s}_{n}\right|^{2}
$$

with $r_{n}$ and $\hat{s}_{n}$ denoting the residual data and the estimate of $s_{n}$, respectively. Figure 9 shows the relation between the estimated frequency and the number $p$ of sinusoids (left panel) and the dependence of the MDL on $p$ (right panel) in the $H$ component at TER in the interval from 15:20:48 UT to 15:25:48 UT on Aug. 13, 1992. The estimated frequencies were relatively stationary over this interval, as indicated later in Fig. 10. The MDL becomes minimum at $p=7$, and it should be noted that the lower estimated frequencies less than $40 \mathrm{mHz}$ change little for $p \geq 7$.
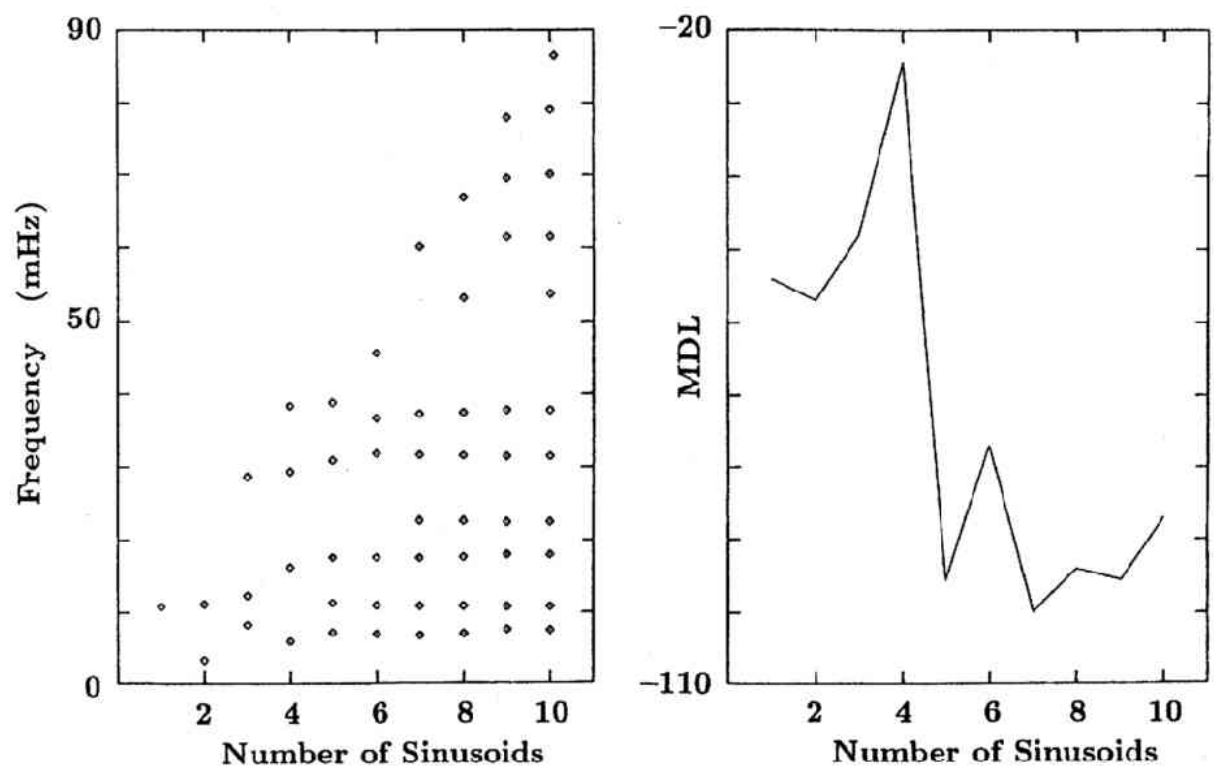

Fig. 9. Relation between the estimated frequency and the number $p$ of sinusoids (left panel) and the dependence of the MDL on $p$ (right panel) in the $H$ component at TER in the interval from 15:20:48 UT to 15:25:48 UT on Aug. 13, 1992. The MDL becomes minimum at $p=7$, and it should be noted that the lower estimated frequencies less than $40 \mathrm{mHz}$ change little for $p \geq 7$. 


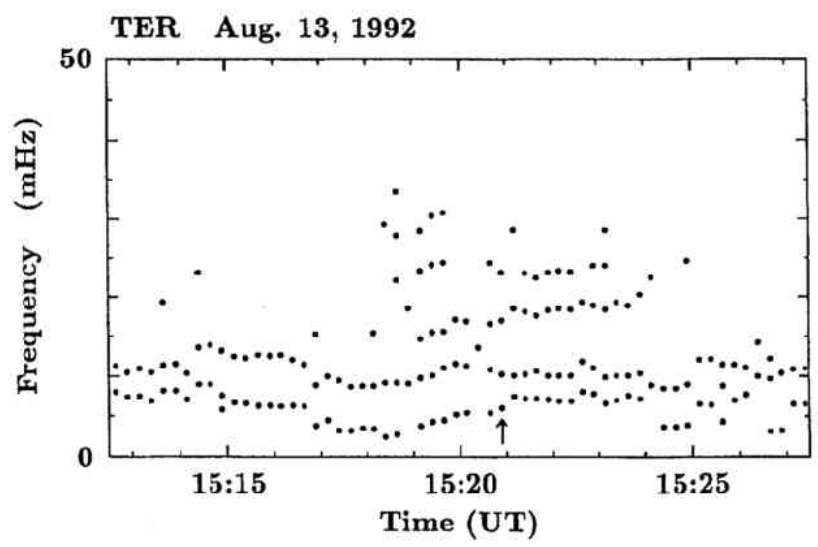

Fig. 10. Temporal variation in frequency of sinusoids in the $H$ component at TER on Aug. 13, 1992. An SSC began at around 15:15 UT and a few minutes later the compressional $\mathrm{HM}$ waves with frequencies greater than $\sim 15 \mathrm{mHz}$ were intensified. The arrow indicates the estimated frequencies in the interval from 15:20:48 UT to 15:25:48 UT.

The sinusoids in each interval have been determined according to the following procedure:

1. Search for such a value of $p$ that the MDL becomes minimum in the range of 1 to 10 .

2. Compute its integrated power given by

$$
P_{k}=\Delta t \sum_{n=0}^{N-1}\left|A_{k} \mathrm{e}^{-\alpha_{k} n \Delta t} \cos \left(\omega_{k} n \Delta t+\phi_{k}\right)\right|^{2}
$$

in each of $p$ sinusoids estimated when the MDL becomes minimum, and denote the maximum value of $P_{k}$ by $P_{\max }$.

3. Select only sinusoids with integrated powers greater than one tenth of $P_{\max }$.

Figure 10 is an example of the temporal variation in frequency of the sinusoids in the $H$ component selected on the procedure. An SSC event began at around 15:15 UT on Aug. 13, 1992 and a few minutes later the compressional HM waves with frequencies greater than $\sim 15 \mathrm{mHz}$ were intensified (Fig. 10). At TER in the interval from 15:20:48 UT to 15:25:48 UT the estimated frequencies of these waves are $17.0 \mathrm{mHz}$ and $23.1 \mathrm{mHz}$ in the $H$ component (cf. Table 3 ). The estimated frequencies in this interval are indicated by the arrow in Fig. 10. Although a frequency of $6.0 \mathrm{mHz}$ is estimated in this interval, it is nearly equal to the cutoff frequency of the PCP filter with $\mu=7$ and so such frequencies are omitted in Tables 3 and 4 . It should be noted that the estimated frequencies are relatively stationary over this interval which was selected for an application example of the method in Fig. 9. While such an interval is also found for the SSC event on Jun. 10, 1992 (e.g., from 04:08:33 UT to 04:13:33 UT), the physical meaning of its presence has been yet unknown.

Listed in Table 3 are the estimated frequencies of $H$ component Psc's in the interval from 15:20:48 UT to 15:25:48 UT on Aug. 13, 1992, and in Table 4 those frequencies in the interval from 04:08:33 UT to $04: 13: 33$ UT on Jun. 10,1992. In these tables the symbol $\times$ means that the integrated power of the component with a corresponding frequency is less in magnitude than a threshold level (one tenth of the maximum value). Since the integrated power is less in magnitude than a threshold level for all corresponding frequencies at KUJ and DAR in case of the SSC event on Aug. 13, 1992, these stations are omitted in Table 3. The frequencies greater than $\sim 15 \mathrm{mHz}$ may correspond to those $(15.5 \mathrm{mHz}$ and 25.3 $\mathrm{mHz}$ ) of the SSC-excited $\mathrm{Pc} 3$ pulsations which were attributed to the cavity-mode oscillations by Yumoto et al. (1992).

As indicated by Tables 3 and 4, the Psc's have globally almost identical dominant frequencies. 
Table 3. Dominant frequencies of $H$ component Psc's in the interval from 15:20:48 UT to 15:25:48 UT on Aug. 13, 1992.

\begin{tabular}{lccr}
\hline Station & \multicolumn{3}{c}{ Frequency $(\mathrm{mHz})$} \\
\hline GUA & 23.3 & 16.6 & 8.7 \\
TAM & $\times$ & 17.5 & 10.3 \\
EUS & 22.4 & 16.7 & 10.3 \\
TER & 23.1 & 17.0 & 10.3 \\
SMA & 23.2 & 17.7 & 10.3 \\
\hline
\end{tabular}

Table 4. Dominant frequencies of $H$ component Psc's in the interval from 04:08:33 UT to 04:13:33 UT on Jun. $10,1992$.

\begin{tabular}{lcccc}
\hline Station & \multicolumn{4}{c}{ Frequency $(\mathrm{mHz})$} \\
\hline KUJ & 28.2 & 19.6 & $\times$ & $\times$ \\
GUA & $\times$ & 21.1 & 15.6 & 8.6 \\
DAR & $\times$ & 19.8 & 16.0 & 9.8 \\
VIC & $\times$ & 22.4 & 14.9 & 9.5 \\
HAR & 28.4 & $\times$ & 14.9 & 7.6 \\
TAM & $\times$ & $\times$ & 14.4 & $\times$ \\
SLZ & $\times$ & $\times$ & 17.3 & 11.2 \\
EUS & 24.3 & $\times$ & 17.9 & 10.4 \\
TER & 24.6 & $\times$ & 17.9 & 10.2 \\
SMA & 25.2 & 19.2 & $\times$ & $\times$ \\
\hline
\end{tabular}

Table 5. Dominant frequencies of $H$ component Pi2's observed at Kuju (KUJ) in Japan, Yap (YAP) in Micronesia, and Maroua (MAR) in Cameroon on Jan. 20, 1991 (after Itonaga and Kitamura (1994)).

\begin{tabular}{lllll}
\hline Station & \multicolumn{4}{c}{ Frequency $(\mathrm{mHz})$} \\
\hline KUJ & 27.7 & 23.0 & 17.3 & 9.4 \\
YAP & 27.4 & 23.3 & 17.3 & 9.3 \\
MAR & 28.3 & 22.9 & 17.8 & 9.1 \\
\hline
\end{tabular}

Further, these frequencies agree well with those of Pi2's (cf. Table 5). This implies that the frequencies of low-latitude and equatorial pulsations may be determined by the global structure of the magnetosphere or the plasmasphere independently of source mechanisms, although the amplitude itself of each frequency component will be dependent on the source mechanism.

\section{Discussion}

The idea of cavity-mode oscillation will be naturally introduced from the conjecture obtained in the last section that the frequencies of low-latitude and equatorial pulsations may be determined by the global structure of the magnetosphere or the plasmasphere. However, the comparison between waveforms indicates that the low-latitude and equatorial Psc's are not cavity-mode oscillations but compressionalHM waves propagating from dayside to nightside along the equatorial plane. Then, if so, it is necessary to examine how such a waveform as could be expressed as a superposition of exponentially damped 
sinusoids, whose frequencies are globally almost identical, is brought about.

In the vicinity of the equatorial plane ( $x-y$ plane) of the magnetosphere, the equations that the electric field $\boldsymbol{E}_{\perp}$ of the HM wave should satisfy become

$$
\begin{gathered}
\left(\nabla^{2}-\frac{1}{V_{A}^{2}} \frac{\partial^{2}}{\partial t^{2}}\right)\left(\nabla \times \boldsymbol{E}_{\perp}\right)_{z}=-\mu_{0}\left(\nabla_{\perp} \ln V_{A}^{2} \times \frac{\partial \boldsymbol{j}_{\perp}}{\partial t}\right)_{z}, \\
\left(\frac{\partial^{2}}{\partial z^{2}}-\frac{1}{V_{A}^{2}} \frac{\partial^{2}}{\partial t^{2}}\right)\left(\nabla \cdot \boldsymbol{E}_{\perp}\right)=-\mu_{0} \nabla_{\perp} \ln V_{A}^{2} \cdot \frac{\partial \boldsymbol{j}_{\perp}}{\partial t},
\end{gathered}
$$

under a cold plasma model (see Appendix), where $\boldsymbol{j}_{\perp}$ is the perpendicular current density, $V_{A}$ the Alfvén velocity, and the $z$ axis is directed along the field line, the direction of which is considered to be almost constant in the vicinity of the equatorial plane. Further, Eqs. (3) and (4) are equivalent to

$$
\begin{gathered}
\left(\nabla^{2}-\frac{1}{V_{A}^{2}} \frac{\partial^{2}}{\partial t^{2}}\right) b_{z}=\mu_{0}\left(\nabla_{\perp} \ln V_{A}^{2} \times j_{\perp}\right)_{z}, \\
\left(\frac{\partial^{2}}{\partial z^{2}}-\frac{1}{V_{A}^{2}} \frac{\partial^{2}}{\partial t^{2}}\right) j_{z}=\nabla_{\perp} \ln V_{A}^{2} \cdot \frac{\partial j_{\perp}}{\partial z},
\end{gathered}
$$

respectively, where $b_{z}$ is the compressional component of the perturbation magnetic field and $j_{z}$ the parallel (field-aligned) current density. In the derivation of Eq. (6), it is assumed that $V_{A}$ is homogeneous along the field line in the vicinity of the equatorial plane.

Now, we consider a situation that the compressional wave with the rotation of the electric field, $(\nabla$ $\left.\times \boldsymbol{E}_{\perp}\right)_{z}$, and the compressional component of the magnetic field, $b_{z}$, propagates two-dimensionally in the vicinity of the equatorial plane with the deformation of wavefront as illustrated in Fig. 10 of Wilken et al. (1982). If $V_{A}$ is homogeneous in the equatorial plane, the waveform of $b_{z}$ only suffers some modification due to a geometric effect according to a spread of the wavefront and is essentially invariant during the propagation of the compressional wave. However, since $V_{A}$ actually has a radial inhomogeneity in the equatorial plane, the perpendicular current $j_{\perp}$ flowing along the wavefront excites the shear Alfvén wave with the divergence of the electric field, $\nabla \cdot \boldsymbol{E}_{\perp}$, and the parallel current, $j_{z}$. Concerning the distribution of $\boldsymbol{j}_{\perp}$ along the field line, we may assume that its magnitude becomes maximum at the equatorial plane. Then, $\nabla \cdot \boldsymbol{E}_{\perp}$ generated via the coupling also has a maximum in magnitude there, as seen from Eq. (4), and this means that the displacement of the field line occurs with its maximum at the equatorial plane. Because of the tension of the field line, such a displacement brings about a local oscillation of the field line in the vicinity of the equatorial plane with its leading edges going away from the plane, and its effect is in turn fed back into the compressional wave. Thus, the waveform of $b_{z}$ can be drastically altered via the coupling between the compressional and shear Alfvén waves. For instance, even if the waveform of $b_{z}$ in the compressional wave generated in the outer magnetosphere has originally no oscillatory structure, it may acquire such a structure owing to the coupling during the passage of the compressional wave within the magnetosphere. In fact, the existence of the oscillations which appear concurrently with MI's of SSC's (cf. Fig. 2) or bay-type disturbances of substorms (e.g., cf. Fig. 4 of Itonaga and Kitamura (1994)) suggests that such a mechanism is promising.

In the outside of the plasmasphere, there exists the coupling between the compressional and shear Alfvén waves in the vicinity of the equatorial plane (cf. Fig. 10 of Wilken et al. (1982)). Such a coupling will be much stronger in the plasmapause region because of a larger gradient of $V_{A}$ than in other regions. 
On the other hand, it will be generally weak within the inner plasmasphere, because the wavefront of the compressional wave tends to surround the earth owing to the slowdown of $V_{A}$ just inside of the plasmapause and so the terms in the righthand side of Eqs. (4) and (6) will be negligibly small within the inner plasmasphere. Then, the waveform of $b_{z}$ in the compressional wave only suffers some modification due to a geometric effect or a diffraction effect there. Thus, during its passage through the plasmapause the compressional HM wave generated in the outer magnetosphere might be regulated in such a waveform as could be expressed as a superposition of exponentially damped sinusoids at ground stations, whose frequencies are globally almost identical. To discuss such a process not speculatively but quantitatively, a reasonable modeling of the magnetosphere and numerical studies based on the model are necded.

A notable PRI appears near the dayside dip equator (at TER and EUS) in the SSC event on Aug. 13, 1992 (cf. Fig. 2). Although it might be possible to consider that the PRI is a part of the Psc, the generation of the PRI would be due to the enhanced ionospheric conductivity at the dayside dip equator. Araki (1977) found a close correlation between PRI's observed at high latitudes and the dayside equator, and concluded that the equatorial PRI is caused by an extension of the ionospheric current responsible for the highlatitude PRI. Kikuchi and Araki (1979b) showed that a horizontal electric field suddenly impressed on the polar ionosphere can be transmitted instantaneously to the equator as the zeroth order TM electromagnetic wave in the earth-ionosphere waveguide. Assuming a realistic conductivity distribution on the spherical thin ionosphere, Tsunomura and Araki (1984) made a static calculation for the global distribution of the ionospheric current produced by the electric field instantaneously transmitted from the polar ionosphere, and found a sharp increase of the westward ionospheric current at the equator enough to explain the dayside enhancement of the PRI. Although the current distribution obtained by them is well consistent with the equivalent current system for the preliminary impulse (PI) of SSC, the apparent phase difference between the PRI's at TER and EUS found in Fig. 5 can be never explained by the PI current system set up with no time delay. When the compressional HM wave is incident on the ionosphere from the magnetosphere, the magnetic field accompanying the $\mathrm{HM}$ wave is prevented from entering the atmosphere by the induced current in the ionosphere and the time delay in its appearance on the ground will be strongly controlled by the ionospheric conductivity determining the intensity of the induced current. Thus, the time delay in the PRI and the subsequent Psc at TER on Aug. 13, 1992, where TER appears to be located nearer the dip equator than EUS (cf. Table 1), implies that the direct interaction between the HM wave and the equatorial ionosphere with the latitudinal inhomogeneity in the conductivity distribution may play a significant role in producing the PRI. It has been shown that it is difficult to produce the PRI by a direct incidence of the plane HM wave on the uniform ionosphere (Kikuchi and Araki, 1979a). However, very little is known about the transient response of the non-uniform equatorial ionosphere to the incident HM wave. A detailed theoretical or numerical research for such a response is a must.

\section{Concluding Remarks}

Using geomagnetic data from a global network equipped with fluxgate magnetometers and data loggers by Kyushu University, we analyzed two SSC events, which occurred on Jun. 10 and Aug. 13, 1992, and examined the global structure of low-latitude and equatorial Psc's. The obtained results are summarized as follows:

1. The shape of of the waveform is consistently ordered by local time for all low-latitude and equatorial stations; it is steeper near noon (or near the source) and has a more moderate slope near midnight (or far from the source). Such a difference in the waveform may be due to a kind of diffraction effect that among compressional HM waves which have entered the plasmasphere, those waves with lower frequencies (or longer wavelengths) propagate around the earth to midnight with less attenuation.

2. The Psc's have globally almost identical dominant frequencies which agree well with those of Pi2's. This implies that the frequencies of low-latitude and equatorial pulsations may be determined by the global structure of the magnetosphere or the plasmasphere independently of source mechanisms, although the amplitude itself of each frequency component will be dependent on the source mechanism. 
It should be noted that these results are by no means conflicting with each other and rather indicate that the power ratio of a frequency component observed at a station near midnight to the corresponding one near noon decreases as the frequency increases. The second result apparently suggests that the idea of cavity-mode oscillation is promising. However, it is concluded from the first result that the low-latitude and equatorial Psc's are not cavity-mode oscillations but compressional HM waves propagating from dayside to nightside two-dimensionally along the equatorial plane. It is thought from a standpoint of propagating wave that during its passage through the plasmapause the compressional HM wave generated in the outer magnetosphere might be regulated in such a waveform as could be expressed as a superposition of exponentially damped sinusoids at ground stations, whose frequencies are globally almost identical. To confirm whether such a regulation is possible or not, further observational and numerical studies are needed.

Further, the equatorial PRI was also discussed. Then, it was suggested from the time delay seen in the equatorial PRI that the direct interaction between compressional HM waves and the non-uniform equatorial ionosphere might play a significant role in producing the PRI.

We said nothing about the Psc's at highest latitude stations (VIC and HAR) and also about their $D$ component variations at low-latitude and equatorial stations. Studies on the global structure of the Psc's including their $D$ component variations are currently in progress with some more high-latitude stations and will appear elsewhere.

The authors are grateful to T. Kikuchi and A. Yoshikawa for their illuminative suggestions to this work. The data used in this work were obtained in cooperation with Guam Observatory (U.S. Geological Survey), Tropical Ecosystems Research Centre (CSIRO, Australia) and CRAAG (Algeria). One of the authors (T. K.) would like to express his sincere thanks to B. J. Fraser, W. F. Stuart and F. X. Ogura for their observational helps and to the Schlumberger K. K. for the financial help to this work. This work was financially supported by the STEP program in Japan, and also supported by The Ministry of Education, Science and Culture under Grants No. 62302018, No. 01044108 , No. 03041061 and No. 05640488.

The Editor thanks T. Araki and M. J. Engebretson for their assistance in evaluating this paper.

\section{APPENDIX}

We derive Eqs. (3) and (4). In a cold magnetized plasma, linearized basic equations are:

$$
\begin{aligned}
& \rho \frac{\partial \boldsymbol{u}_{\perp}}{\partial t}=\boldsymbol{j}_{\perp} \times \boldsymbol{B}, \\
& \nabla \times \boldsymbol{b}=\mu_{0} \boldsymbol{j}, \\
& \nabla \times \boldsymbol{E}_{\perp}=-\frac{\partial \boldsymbol{b}}{\partial t}, \\
& \boldsymbol{E}_{\perp}+\boldsymbol{u}_{\perp} \times \boldsymbol{B}=0,
\end{aligned}
$$

where $\mu_{0}$ is the magnetic permeability of vacuum, $\rho$ the unperturbed plasma density, $\boldsymbol{B}$ the ambient magnetic field, and $\boldsymbol{u}, \boldsymbol{j}, \boldsymbol{E}$ and $\boldsymbol{b}$ the perturbation vector quantities (velocity, current density, electric field and magnetic field) with the subscript $\perp$ denoting the part perpendicular to $\boldsymbol{B}$. We consider a situation that while $\boldsymbol{B}$ is constant and parallel to the $z$ axis, $\rho$ has an inhomogeneous distribution.

Taking the rotation of Eq. (A4), we have

$$
\left(\nabla \times E_{\perp}\right)_{z}=B \nabla \cdot \boldsymbol{u}_{\perp}
$$


and so

$$
\frac{\partial}{\partial t}\left(\nabla \times \boldsymbol{E}_{\perp}\right)_{z}=\frac{B^{2}}{\rho}(\nabla \times \boldsymbol{j})_{z}+\left[\nabla_{\perp}\left(\frac{B^{2}}{\rho}\right) \times \boldsymbol{j}_{\perp}\right]_{z}
$$

using Eq. (A1), where $B=|B|$ and the subscript $z$ denotes the $z$ component. The equations

$$
\nabla \cdot\left(\boldsymbol{j}_{\perp} \times \boldsymbol{B}\right)=B(\nabla \times \boldsymbol{j})_{z}, \quad \nabla_{\perp}\left(\frac{B}{\rho}\right) \cdot\left(\boldsymbol{j}_{\perp} \times \boldsymbol{B}\right)=B\left[\nabla_{\perp}\left(\frac{B}{\rho}\right) \times \boldsymbol{j}_{\perp}\right]_{z}
$$

are also utilized in the derivation of Eq. (A5). Further, by letting

$$
V_{A}=\frac{B}{\sqrt{\mu_{0} \rho}}
$$

and using Eq. (A2) and the equation

$$
[\nabla \times(\nabla \times b)]_{z}=-\nabla^{2} b_{z}
$$

Eq. (A5) becomes

$$
\frac{\partial}{\partial t}\left(\nabla \times \boldsymbol{E}_{\perp}\right)_{z}=-V_{A}^{2} \nabla^{2} b_{z}+\mu_{0}\left(\nabla_{\perp} V_{A}^{2} \times \boldsymbol{j}_{\perp}\right)_{z}
$$

Then, considering Eq. (A3), we obtain

$$
\frac{\partial^{2}}{\partial t^{2}}\left(\nabla \times E_{\perp}\right)_{z}=V_{A}^{2} \nabla^{2}\left(\nabla \times E_{\perp}\right)_{z}+\mu_{0}\left(\nabla_{\perp} V_{A}^{2} \times \frac{\partial j_{\perp}}{\partial t}\right)_{z}
$$

from Eq. (A6).

Next, taking the divergence of Eq. (A4), we have

$$
\nabla \cdot \boldsymbol{E}_{\perp}=-B\left(\nabla \times \boldsymbol{u}_{\perp}\right)_{z},
$$

and so

$$
\frac{\partial}{\partial t}\left(\nabla \cdot \boldsymbol{E}_{\perp}\right)=V_{A}^{2} \nabla \cdot(\nabla \times \boldsymbol{b})_{\perp}+\mu_{0} \nabla_{\perp} V_{A}^{2} \cdot \boldsymbol{j}_{\perp}
$$

using Eqs. (A1) and (A2). The equations

$$
\left[\nabla \times\left(\boldsymbol{j}_{\perp} \times \boldsymbol{B}\right)\right]_{z}=-B \nabla \cdot \boldsymbol{j}_{\perp},\left[\nabla_{\perp}\left(\frac{B}{\rho}\right) \times\left(\boldsymbol{j}_{\perp} \times \boldsymbol{B}\right)\right]_{z}=-B \nabla_{\perp}\left(\frac{B}{\rho}\right) \cdot \boldsymbol{j}_{\perp}
$$


are also utilized in the derivation of Eq. (A8). Considering Eq. (A3), we obtain

$$
\frac{\partial^{2}}{\partial t^{2}}\left(\nabla \cdot \boldsymbol{E}_{\perp}\right)=-V_{A}^{2} \nabla \cdot\left[\nabla \times\left(\nabla \times \boldsymbol{E}_{\perp}\right)\right]_{\perp}+\mu_{0} \nabla_{\perp} V_{A}^{2} \cdot \frac{\partial \boldsymbol{j}_{\perp}}{\partial t}
$$

from Eq. (A8). Here, note that

$$
\left[\nabla \times\left(\nabla \times \boldsymbol{E}_{\perp}\right)\right]_{z}=\frac{\partial}{\partial z}\left(\nabla \cdot \boldsymbol{E}_{\perp}\right) .
$$

Then, since

$$
\begin{aligned}
0 & =\nabla \cdot\left[\nabla \times\left(\nabla \times \boldsymbol{E}_{\perp}\right)\right] \\
& =\nabla \cdot\left[\nabla \times\left(\nabla \times \boldsymbol{E}_{\perp}\right)\right]_{\perp}+\frac{\partial^{2}}{\partial z^{2}}\left(\nabla \cdot \boldsymbol{E}_{\perp}\right),
\end{aligned}
$$

Eq. (A9) results in

$$
\frac{\partial^{2}}{\partial t^{2}}\left(\nabla \cdot \boldsymbol{E}_{\perp}\right)=V_{A}^{2} \frac{\partial^{2}}{\partial z^{2}}\left(\nabla \cdot E_{\perp}\right)+\mu_{0} \nabla_{\perp} V_{A}^{2} \cdot \frac{\partial j_{\perp}}{\partial t} .
$$

Equations (3) and (4) are readily derived from Eqs. (A7) and (A10), respectively. In the vicinity of the equatorial plane $(x-y$ plane) of the magnetosphere, the direction of the ambient magnetic field $\boldsymbol{B}$ is considered to be almost constant, while its strength $B$ is radially inhomogeneous. Then, strictly speaking, Eqs. (3) and (4) can not be justified even in the vicinity of the equatorial plane because the constant $B$ is assumed in their derivation. In the plasmapause region and within the inner plasmasphere, however, these equations will approximately hold in the vicinity of the equatorial plane because $B$ is considered to have a more moderate radial variation than the plasma density $\rho$.

Since

$$
-\frac{\partial b_{z}}{\partial t}=\left(\nabla \times \boldsymbol{E}_{\perp}\right)_{z}
$$

from Eq. (A3), Eq. (3) is equivalent to Eq. (5). Further, from Eqs. (A2) and (A3) we have

$$
\mu_{0} \frac{\partial j_{z}}{\partial t}=-\frac{\partial}{\partial z}\left(\nabla \cdot E_{\perp}\right)
$$

Then, if $V_{A}$ is homogeneous along the $z$ axis, Eq. (4) is also equivalent to Eq. (6).

\section{REFERENCES}

Araki, T., Global structure of geomagnetic sudden commencements, Planet. Space Sci., 25, 373-384, 1977.

Itonaga, M., Parameter estimation of exponentially damped sinusoids using a modified Pisarenko method, Technol. Rep. Kyushu Univ., 63, 567-571, 1990 (in Japanese with English abstract).

Itonaga, M., Nonlinear filtering using a piecewise cubic polynomial, submitted to Trans. Japan SIAM, 1995 (in Japanese with English abstract). 
Itonaga, M. and T.-I. Kitamura, Smoothing of geomagnetic data using a piecewise cubic polynomial, Geophys. J. Int., 116, 655$662,1994$.

Kaufmann, R. L. and D. N. Walker, Hydromagnetic waves excited during an SSC, J. Geophys. Res., 79, 5187-5195, 1974.

Kikuchi, T. and T. Araki, Transient response of uniform ionosphere and preliminary reverse impulse of geomagnetic storm sudden commencement, J. Atmos. Terr. Phys., 41, 917-925, 1979a.

Kikuchi, T. and T. Araki, Horizontal transmission of the polar electric field to the equator, J. Atmos. Terr. Phys., 41, 927-936, 1979b.

Kivelson, M. G. and D. J. Southwood, Resonant ULF waves: A new interpretation, Geophys. Res. Lett., 12, 49-52, 1985.

Kivelson, M. G. and D. J. Southwood, Coupling of global magnetospheric MHD eigenmodes to field line resonances, J. Geophys. Res., 91, 4345-4351, 1986.

Ohnishi, H. and T. Araki, Two-dimensional interaction between a plane hydromagnetic wave and the earth-ionosphere system with curvature, Ann. Geophysicae, 10, 281-287, 1992.

Rastogi, R. G., Longitudinal variation of sudden commencement of geomagnetic storm at equatorial stations, J. Geophys. Res., 98, 15,411-15,416, 1993.

Rissancn, J., Modeling by shortest data description, Automatica, 14, 465-471, 1978.

Saito, T. and S. Matsushita, Geomagnetic pulsations associated with sudden commencements and sudden impulses, Planet. Space Sci., 15, 573-587, 1967.

Tsunomura, S. and T. Araki, Numerical analysis of equatorial enhancement of geomagnetic sudden commencement, Planet. Space Sci., 32, 599-604, 1984.

Wedeken, U., H. Voelker, K. Knott, and M. Lester, SSC-excited pulsations recorded near noon on GEOS 2 and on the ground (CDAW 6), J. Geophys. Res., 91, 3089-3100, 1986.

Wilken, B., C. K. Goertz, D. N. Baker, P. R. Higbie, and T. A. Fritz, The SSC on July 29, 1977 and its propagation within the magnetosphere, J. Geophys. Res., 87, 5901-5910, 1982.

Yumoto, K., Y. Tanaka, T. Oguti, K. Shiokawa, Y. Yoshimura, A. Isono, B. J. Fraser, F. W. Menk, J. W. Lynn, M. Seto, and $210^{\circ}$ MM Magnetic Observation Group, Globally coordinated magnetic observations along $210^{\circ}$ magnetic meridian during STEP period: 1. Preliminary results of low-latitude Pc 3's, J. Geomag. Geoelectr., 44, 261-276, 1992. 